\title{
Compressional Alfvén eigenmode structure in spherical tokamaks
}

\author{
H M Smith $\ddagger$ and E Verwichte \\ Centre for Fusion, Space and Astrophysics, University of Warwick, Coventry CV4 \\ 7AL, United Kingdom \\ E-mail: hakan.smith@warwick.ac.uk
}

\begin{abstract}
The two-dimensional structure of compressional Alfvén eigenmodes below the ion cyclotron frequency is studied numerically by solving the cold plasma Hall-MHD equations for a realistic spherical tokamak equilibrium. The simplest of the computed eigenmodes have a standing wave-like structure and the higher frequency solutions show a more travelling wave-like behaviour. The effects of the equilibrium current and the Hall terms in the eigenmode equation are investigated, and these terms are found to shift the frequencies of eigenmodes with different sign of the toroidal mode number away from each other. A classification scheme is proposed which relates each spherical tokamak eigenmode to the three mode numbers of the corresponding large aspect ratio circular cross section eigenmode obtained by gradually decreasing the elongation and increasing the aspect ratio.
\end{abstract}

PACS numbers: 52.35.Bj, 52.35.Hr, 52.55.Fa, 51.10.+y

$\ddagger$ Present address: Max-Planck-Institut für Plasmaphysik, EURATOM-Association, D-17491, Greifswald, Germany 


\section{Introduction}

Compressional Alfvén eigenmodes (CAEs) have been extensively studied, because they could potentially be applied to channel energy from the fusion products to the background ions in a future reactor or to diagnose fast ion distributions or bulk plasma properties [1]. A theory for CAEs was first developed for large aspect ratio, cylindrical geometry in $[2,3]$, where it was found that the modes can be radially localized near the edge. This theory explained the observations of emission of radiation at harmonics of the ion cyclotron frequency caused by super-Alfvénic ions [4, 5]. Furthermore, the modes were predicted to be localized poloidally as well as radially $[6,7]$ and the Hall term was seen to introduce a dependence of the eigenmode solutions on the poloidal phase velocity $[8,9,10]$.

Super-Alfvénic ions due to neutral beam injection have been observed to generate magnetic fluctuations in the frequency range $0.3 \lesssim \omega / \omega_{\text {ci }} \lesssim 1$, where $\omega_{\text {ci }}$ is the on-axis ion cyclotron frequency, in the spherical tokamaks START [11], NSTX [12] and MAST $[13,14]$ and in the conventional tokamak DIII-D [15]. These perturbations were found in a series of papers $[16,17,18,19,20,14,15]$ to be CAEs and in some cases global Alfvén eigenmodes (GAEs) [21] driven by the energetic ions. For the instabilities identified as CAEs the instability frequency correlates with the evolution of the Alfvén velocity [12] and the perturbed magnetic field polarization is elliptical with a significant component parallel to the equilibrium field $[13,14,19]$. The theories developed for large aspect ratio tokamaks were extended in $[17,20]$ to explain the observations below the ion cyclotron frequency in spherical tokamaks. These analytical works, however, suffered from limiting assumptions that were necessary in order to make the problem tractable. Furthermore, the numerical approaches that have been employed to make more detailed calculations have either not been suitable for large ellipticity tight aspect ratio plasmas [22], or do not include the Hall term [23].

In this paper, we investigate the frequencies and spatial structures of CAEs in spherical tokamaks. The two-dimensional structure of the eigenmodes is studied by deriving the cold plasma Hall-MHD equations for a realistic spherical tokamak equilibrium and solving them using a finite difference scheme. The cold plasma assumption excludes the slow magnetoacoustic mode from the problem. The parallel wavenumber $k_{\|}$is allowed to be non-zero, but assumed to be small enough $\left(k_{\|}^{2} \ll \omega^{2} / v_{\mathrm{A}}^{2}\right)$ to avoid coupling to the shear Alfvén branch. For a spherical tokamak it is important to correctly model the equilibrium magnetic field, which is therefore obtained from the equilibrium solver CHEASE [24]. The equilibrium current is also retained in the linearized Hall-MHD equations, and the influence of both the Hall term and the equilibrium current on the eigenmode frequency is studied.

The structure of the paper is the following: In Sec. 2 the eigenmode equations are derived. To solve them, the numerical solution scheme described in Sec. 3 is used with the boundary conditions in Sec. 4 . The calculated eigenmodes and eigenfrequencies are presented and discussed in comparison with experiments and other theoretical results 
in Sec. 5. Finally, the main conclusions are given in Sec. 6 .

\section{The eigenmode equation}

The starting point of the analysis is the linearized momentum balance equation

$$
-\mathrm{i} \omega \rho \boldsymbol{v}=\boldsymbol{J}_{1} \times \boldsymbol{B}_{0}+\boldsymbol{J}_{0} \times \boldsymbol{B}_{1},
$$

and the linearized Ohms law

$$
\boldsymbol{E}=\left(\frac{\boldsymbol{J}_{1}}{n_{\mathrm{e}} e}-\boldsymbol{v}\right) \times \boldsymbol{B}_{0}+\frac{\boldsymbol{J}_{0}}{n_{\mathrm{e}} e} \times \boldsymbol{B}_{1}
$$

where $\boldsymbol{J}$ is current density, $\boldsymbol{B}$ and $\boldsymbol{E}$ are magnetic and electric fields, $\boldsymbol{v}$ is velocity, $\rho$ is mass density, $n_{\mathrm{e}}$ is electron density and $e$ is the elementary charge. Equilibrium quantities are denoted by the subscript 0 and perturbed quantities, which vary as $\exp (-\mathrm{i} \omega t)$, are denoted by a subscript 1 . Together with the Maxwell equations, where the displacement current is neglected, the relations (1) and (2) yield a wave equation which can be written either for the perturbed electric field $\boldsymbol{E}$ or perturbed magnetic field $\boldsymbol{B}_{1}$,

$\omega^{2} \boldsymbol{E}=\mathrm{i} F(\nabla \times \nabla \times \boldsymbol{E}) \times \boldsymbol{B}_{0}+G B_{0}^{2}(\nabla \times \nabla \times \boldsymbol{E})_{\perp}-G\left(\tilde{\boldsymbol{J}}_{0} \times \nabla \times \boldsymbol{E}\right) \times \boldsymbol{B}_{0}+\mathrm{i} F \tilde{\boldsymbol{J}}_{0} \times \nabla \times \boldsymbol{E},(3)$

$\omega^{2} \boldsymbol{B}_{1}=\nabla \times\left\{\left[\mathrm{i} F \nabla \times \boldsymbol{B}_{1}-G\left(\nabla \times \boldsymbol{B}_{1}\right) \times \boldsymbol{B}_{0}-G \tilde{\boldsymbol{J}}_{0} \times \boldsymbol{B}_{1}\right] \times \boldsymbol{B}_{0}+\mathrm{i} F \tilde{\boldsymbol{J}}_{0} \times \boldsymbol{B}_{1}\right\}$.

Here, $F=-\omega v_{\mathrm{A}}^{2} /\left(\omega_{\mathrm{ci}} B_{0}\right), G=v_{\mathrm{A}}^{2} / B_{0}^{2}, \omega_{\text {ci }}$ is the ion cyclotron frequency, $v_{\mathrm{A}}$ is the Alfvén velocity, $\tilde{\boldsymbol{J}}_{0} \equiv \mu_{0} \boldsymbol{J}_{0}=\nabla \times \boldsymbol{B}_{0}$ is the equilibrium current, and the subscript $\perp$ should be interpreted as $\mathbf{X}_{\perp}=-B_{0}^{-2} \boldsymbol{B}_{0} \times\left(\boldsymbol{B}_{0} \times \mathbf{X}\right)$.

A usual approach [25] is to choose (3) as the main equation, neglect all $\tilde{\boldsymbol{J}}_{0}$ terms, and transform it into the form

$$
\frac{c^{2}}{\omega^{2}} \nabla \times \nabla \times \boldsymbol{E}_{1}=\epsilon_{1} \boldsymbol{E}_{1 \perp}+\mathrm{i} \epsilon_{2} \boldsymbol{E}_{1} \times \boldsymbol{B}_{0} / B_{0},
$$

where the dielectric tensor elements $\epsilon_{1}$ and $\epsilon_{2}$ are related to $F$ and $G$ by

$$
\begin{aligned}
& F=\frac{-\varepsilon_{2} c^{2}}{B_{0}\left(\varepsilon_{1}^{2}+\varepsilon_{2}^{2}\right)} \\
& G=\frac{\varepsilon_{1} c^{2}}{B_{0}^{2}\left(\varepsilon_{1}^{2}+\varepsilon_{2}^{2}\right)} .
\end{aligned}
$$

On the outboard side of a tight aspect ratio tokamak like MAST the equilibrium magnetic field is far from homogeneous, so $\tilde{\boldsymbol{J}}_{0}$ cannot in general be neglected. Equation (5) can therefore be modified to account for the $\tilde{\boldsymbol{J}}_{0}$ terms [26, 27], or one can work with (3) or (4) directly. In the present work it is preferred to solve (4) for the magnetic field, because the compressional wave has for perpendicular propagation one dominant perturbed magnetic field component, i.e. the parallel component.

The perturbed magnetic field $\boldsymbol{B}_{1}$ is represented as the sum of three orthogonal components,

$$
\boldsymbol{B}_{1}=b_{r} \nabla r+b_{\wedge} \boldsymbol{\Lambda}+b_{\|} \boldsymbol{B}_{0},
$$


where $r$ is a flux surface label and $\boldsymbol{\Lambda}=\boldsymbol{B}_{0} \times \nabla r$. The three functions $b_{\|}, b_{r}$, and $b_{\wedge}$ are governed by the three components of (4) along $\nabla r, \boldsymbol{\Lambda}$ and $\boldsymbol{B}_{0}$ supplemented by appropriate boundary conditions at the edge of the plasma. For the presentation of results in Sec. 5, the more physically relevant quantities $B_{r}=b_{r} \sqrt{g^{r r}}, B_{\wedge}=b_{\wedge} \sqrt{g^{r r}} B_{0}$ and $B_{\|}=b_{\|} B_{0}$ will be used, where $g^{r r}=\nabla r \cdot \nabla r$.

The equations are simplified by assuming that $k_{\|}^{2} \ll \omega^{2} / v_{\mathrm{A}}^{2}$, which written in operator form becomes

$$
\omega^{2} X \gg \frac{v_{\mathrm{A}}^{2}}{B_{0}^{2}}\left(\boldsymbol{B}_{0} \cdot \nabla\right)^{2} X
$$

where $X$ is a perturbed quantity. This approximation is necessary to exclude shear wave (i.e. ion cyclotron mode) perturbations, which are difficult to treat with the numerical scheme described below. It can be verified a posteriori that the obtained eigenmodes do not satisfy the $\epsilon_{1}=c^{2} k_{\|}^{2} / \omega^{2}$ singularity of cold MHD in the region of their localization. Furthermore, because of toroidal symmetry a single toroidal mode number $n$ is considered, so that $X$ varies as $\exp (-\mathrm{i} n \varphi-\mathrm{i} \omega t)$, which essentially reduces the problem to two dimensions, radial and poloidal.

The full equations, i.e. the three components of (4), are not shown here because of their length but are given in (A.1) to (A.3) in the Appendix. These are the equations that, together with the assumption (9), are solved in the numerical calculation. However, for the purpose of the simplicity of this presentation, we may assume that $\boldsymbol{J}_{0}=\mathbf{0}$ and $\left(\boldsymbol{B}_{0} \cdot \nabla\right) \nabla r=\mathbf{0}$ and that the spatial variation along $\boldsymbol{B}_{0}$ of equilibrium quantities occurs on longer scales than that of perturbed quantities, in which case the three components of (4) reduce to

$$
\begin{aligned}
& \omega^{2} g^{r r} b_{r}=\boldsymbol{B}_{0} \cdot \nabla\left[\mathrm{i} F \boldsymbol{\Lambda} \cdot \nabla b_{\|}+G B_{0}^{2} \nabla r \cdot \nabla b_{\|}\right], \\
& \omega^{2} g^{r r} B_{0}^{2} b_{\wedge}=\boldsymbol{B}_{0} \cdot \nabla\left[-\mathrm{i} F B_{0}^{2} \nabla r \cdot \nabla b_{\|}+G B_{0}^{2} \boldsymbol{\Lambda} \cdot \nabla b_{\|}\right], \\
& \begin{aligned}
\omega^{2} B_{0}^{2} b_{\|}=\nabla \cdot & \left\{\mathrm{i} F\left[B_{0}^{2} \boldsymbol{B}_{0} \times \nabla b_{\|}-\boldsymbol{\Lambda}\left(\boldsymbol{B}_{0} \cdot \nabla\right) b_{r}+B_{0}^{2} \nabla r\left(\boldsymbol{B}_{0} \cdot \nabla\right) b_{\wedge}\right]+\right. \\
& \left.+G B_{0}^{2}\left[-B_{0}^{2} \nabla_{\perp} b_{\|}+\nabla r\left(\boldsymbol{B}_{0} \cdot \nabla\right) b_{r}+\boldsymbol{\Lambda}\left(\boldsymbol{B}_{0} \cdot \nabla\right) b_{\wedge}\right]\right\} .
\end{aligned}
\end{aligned}
$$

In these simplified equations, a few important things should be noted which are also valid for the full problem. Firstly, all terms including the small $\left(\boldsymbol{B}_{0} \cdot \nabla\right)^{2}$ operator acting on $b_{r}$ and $b_{\wedge}$ have been neglected in (10) and (11). What remains relates $b_{r}$ and $b_{\wedge}$ to $b_{\|}$through a single $\left(\boldsymbol{B}_{0} \cdot \nabla\right)$ operator, so the perpendicular magnetic field components of the solution are small compared with the parallel component. Secondly, the apparent option to substitute (10) and (11) into (12) to reduce the system to one equation becomes impossible for the full system (A.1) - (A.3). However, it can be performed for plane waves in the limit of straight magnetic field and small $k_{\|}$, and the result is the dispersion relation $\Omega_{\mathrm{i}}^{2} \omega^{2}\left(\omega^{2}-v_{\mathrm{A}}^{2} k_{\perp}^{2}\right)=\left(\omega^{2}+\Omega_{\mathrm{i}}^{2}\right) v_{\mathrm{A}}^{4} k_{\perp}^{2} k_{\|}^{2}$, where $k_{\perp}$ is the perpendicular wavenumber (see e.g. [28]). In this context, since the two equations (10) and (11) were substituted into (12), the assumption (9) implies that terms of the order $\Omega_{\mathrm{i}}^{2} v_{\mathrm{A}}^{4} k_{\|}^{4}$ have been neglected in the dispersion relation. 


\section{Numerical solution}

The eigenmodes are expected to be localized to the outboard side of the tokamak and contain only a few wavelengths in the poloidal direction $[17,20,23]$. We choose to discretize the poloidal plane in the two spacial variables $r$ and $\theta$, rather than in $r$ and in Fourier harmonics of $\theta$ as in [23]. For simplicity, finite differences are used to discretize the eigenmode equations on a uniform grid of the coordinates $r$ and $\theta$ with the step sizes $\Delta r$ and $\Delta \theta$. The functions $r=r(\Psi)$ and $\theta=\theta(\Psi, \chi)$ relate $r$ and $\theta$ to the the poloidal flux $\Psi$ and the poloidal coordinate $\chi$ obtained from an equilibrium solver. The coordinates $r, \theta, \Psi$ and $\chi$ all lie in the plane perpendicular to $\nabla \phi$, where $\phi$ is the ordinary toroidal angle. The two coordinates $r$ and $\theta$ are chosen in order for the numerical scheme to work well, and are in general not straight field line coordinates.

For the discretization to be suitable for the finite difference scheme, it is desirable that the grid elements are as square-shaped as possible. In order for them not to be stretched out poloidally, the number of poloidal grid points $N_{\theta}$ is chosen to be higher than the radial number $N_{r}\left(N_{\theta}=160\right.$ and $N_{r}=60$ in the examples below). The transformation functions $r=r(\Psi)$ and $\theta=\theta(\Psi, \chi)$ are chosen to make the skewness of the grid elements small. Combinations of elementary functions are used, to ensure that no numerical derivatives are needed in order to derive the metric tensor elements for $r, \theta, \phi$ from the metric tensor elements of the $\Psi, \chi, \phi$ system known from the equilibrium solver,

$$
\begin{aligned}
g^{r r} & =\nabla r \cdot \nabla r=\left(\frac{\mathrm{d} r}{\mathrm{~d} \Psi}\right)^{2} g^{\Psi \Psi} \\
g^{r \theta} & =\nabla r \cdot \nabla \theta=\frac{\mathrm{d} r}{\mathrm{~d} \Psi}\left(\frac{\partial \theta}{\partial \Psi} g^{\Psi \Psi}+\frac{\partial \theta}{\partial \chi} g^{\Psi \chi}\right) \\
g^{\theta \theta} & =\nabla \theta \cdot \nabla \theta=\left(\frac{\partial \theta}{\partial \Psi}\right)^{2} g^{\Psi \Psi}+2 \frac{\partial \theta}{\partial \Psi} \frac{\partial \theta}{\partial \chi} g^{\Psi \chi}+\left(\frac{\partial \theta}{\partial \chi}\right)^{2} g^{\chi \chi} \\
g^{\phi \phi} & =\nabla \phi \cdot \nabla \phi=R^{-2}
\end{aligned}
$$

where $R$ is the major radius. To avoid numerical difficulties associated with the high Alfvén velocity near the inboard edge (further discussed below) and the geometry near the x-point, and to decrease the computation load, the calculation domain is restricted to be inside of the $\Psi=0.8 \Psi_{\text {separatrix }}$ surface. That the reduction of the computational area only has a small effect on the result is discussed further in Section 4. Figure 1 shows examples of a grid from the equilibrium solver CHEASE [24], and a grid used for the numerical solution of the eigenmode equations.

The equilibrium quantities (magnetic field, current, etc.) are interpolated to the new grid using piecewise cubic splines. This gives small numerical errors, which can however become important when derivatives of the equilibrium quantities are needed. For instance, to calculate $\left(\boldsymbol{B}_{0} \cdot \nabla\right) \nabla r$, which appears in several terms in (A.1) - (A.3), one needs to take derivatives of metric tensor elements. In this case it is necessary to perform a Gaussian smoothing of the numerical derivatives before giving $\left(\boldsymbol{B}_{0} \cdot \nabla\right) \nabla r$ as 

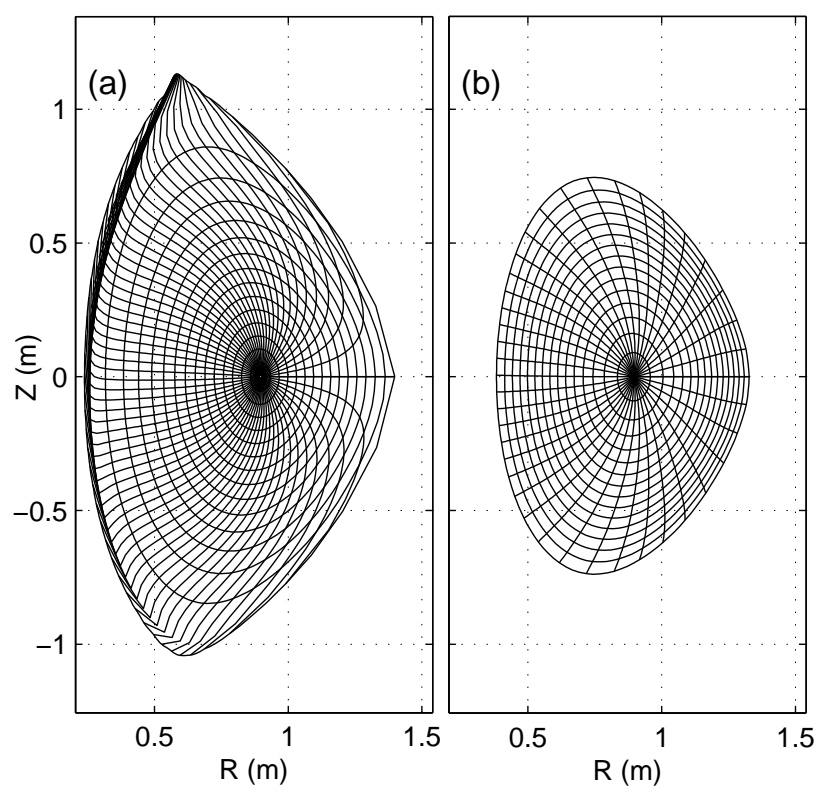

Figure 1. (a) The $(\Psi, \chi)$ grid obtained from the equilibrium solver. (b) The more regular $(r, \theta)$ grid used in the eigenmode calculations. Every fourth grid point in each direction is displayed.

input to the eigenmode calculation.

The eigenmode equations can be rewritten in the general form

$\omega^{2} b_{j}=\sum_{k=r, \wedge, \|} C_{j k}^{(0)} b_{k}+C_{j k}^{(r)} \frac{\partial b_{k}}{\partial r}+C_{j k}^{(\theta)} \frac{\partial b_{k}}{\partial \theta}+C_{j k}^{(r r)} \frac{\partial^{2} b_{k}}{\partial r^{2}}+C_{j k}^{(r \theta)} \frac{\partial^{2} b_{k}}{\partial r \partial \theta}+C_{j k}^{(\theta \theta)} \frac{\partial^{2} b_{k}}{\partial \theta^{2}}$,

for $j=r, \wedge, \|$, where the $C_{j k}$ coefficients are derived from (A.1) - (A.3). By discretizing the partial derivatives, one can write the eigenmode equation as

$$
\omega^{2} b_{j, i_{r}, i_{\theta}}=\sum_{k=r, \wedge, \|} \sum_{d=0, r, \theta, r r, r \theta, \theta \theta} C_{j k}^{(d)} \sum_{l, m} \delta_{l, m}^{(d)} b_{k, i_{r}+l, i_{\theta}+m},
$$

where $b_{k, i_{r}, i_{\theta}}$ is the $k$-component of the magnetic field at the radial grid point $i_{r}$ and poloidal grid point $i_{\theta}$. The non-zero partial derivative discretization coefficients $\delta_{l, m}^{(d)}$ used here are

$$
\begin{aligned}
& \delta_{1,1}^{(r)}=\delta_{1,-1}^{(r)}=-\delta_{-1,1}^{(r)}=-\delta_{-1,-1}^{(r)}=1 / 8 \Delta r, \\
& \delta_{1,0}^{(r)}=-\delta_{-1,0}^{(r)}=1 / 4 \Delta r, \\
& \delta_{0,0}^{(r r)}=-1 /(\Delta r)^{2}, \\
& \delta_{1,0}^{(r r)}=\delta_{-1,0}^{(r r)}=-\delta_{0,1}^{(r r)}=-\delta_{0,-1}^{(r r)}=1 / 2(\Delta r)^{2}, \\
& \delta_{1,1}^{(r r)}=\delta_{1,-1}^{(r r)}=\delta_{-1,1}^{(r r)}=\delta_{-1,-1}^{(r r)}=1 / 4(\Delta r)^{2}, \\
& \delta_{1,1}^{(r \theta)}=\delta_{-1,-1}^{(r \theta)}=-\delta_{1,-1}^{(r \theta)}=-\delta_{-1,1}^{(r \theta)}=1 / 4 \Delta r \Delta \theta, \\
& \delta_{l, m}^{(\theta)}=\delta_{m, l}^{(r)}, \quad \delta_{l, m}^{(\theta \theta)}=\delta_{l, m}^{(r r)},
\end{aligned}
$$

which include a weight spreading in the non-derivative direction to suppress short wavelength noise on the scale of the grid. These numerical problems occur for tight 


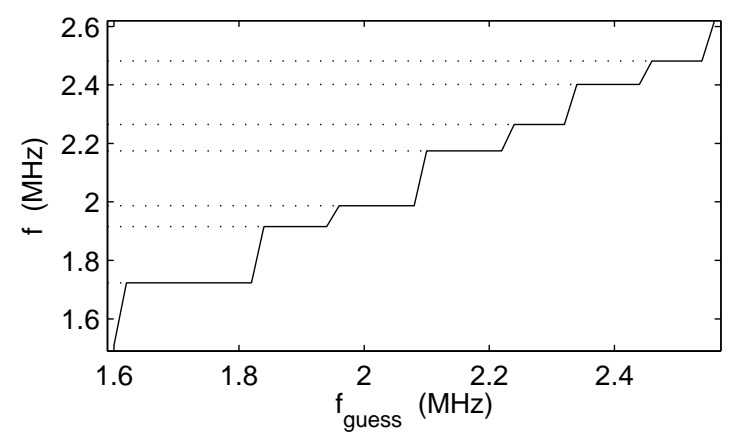

Figure 2. The eigenfrequency versus the initial guess after two iterations with the eigenvalue solver.

aspect ratio configurations near the inboard edge, where the high magnetic field and the low density makes $v_{\mathrm{A}}$ much greater than in the rest of the domain. For the MAST equilibrium the weight spreading introduced above is not enough to resolve the problem. Instead of resorting to more advanced numerical techniques, an artificially high density will be used at the inboard edge. Because the eigenmodes are localised on the outboard side, this has a very small effect on the result; a doubling of the inboard edge density gives only a few percent change in the eigenmode frequency.

For the numerical solution, (15) is turned into a sparse matrix equation $\omega^{2} B b=A b$. All the grid points are numbered with a single index from 1 to $L=N_{r} N_{\theta}+1$ (where the +1 accounts for the point at the magnetic axis, see Sec. 4) and the elements of the eigenvector can thus be numbered by an index from 1 to $3 L$. The $3 L \times 3 L$ banded matrix $A$ corresponds to the right hand side of (15). The diagonal matrix $B$ has ones on the diagonal except for the lines corresponding to equations for boundary points, where the boundary condition may require the left hand side to be zero.

Since the Hall term coefficient $F$ contains $\omega$, so does the right hand sides of (4) and (14). The equation $\omega^{2} B b=A b$ can therefore not be solved as an eigenvalue problem for $\omega^{2}$ straight away. Instead, the terms including $F$ in the equation are refined iteratively. First, a starting guess $\omega_{\text {guess }}$ is made, and the corresponding $F_{\text {guess }}$ is calculated. Next, the eigenvalue problem is solved numerically to obtain $\omega$, which is then used as a new $\omega_{\text {guess }}$, and the process is repeated. The numerical solution is obtained using ARPACK routines [29] which find the eigenvalue that is closest to a given input value. This initial guess for the eigenvalue is taken to be the same as the starting guess $\omega_{\text {guess }}$ in the Hall term. The iterative process of putting the obtained eigenvalue as a new $\omega_{\text {guess }}$ and solving the eigenvalue problem again converges in a few steps if the initial guess is close to an eigenvalue. To find all relevant eigenmodes in a certain frequency span, the starting guess $\omega_{\text {guess }}$ is scanned through this range of frequencies. Figure 2 shows an example of MAST calculations for $n=6$, where the obtained eigenfrequencies $f$ are plotted against $f_{\text {guess }}$. The plateaus identify the true eigenfrequencies. 


\section{Boundary conditions}

Boundary conditions are needed for the perturbed field at the plasma edge, i.e. near the last closed flux surface where the plasma density is low. The most realistic choice would be to use plasma - vacuum boundary conditions, which say that the components of $\boldsymbol{B}_{1}$ parallel to $\boldsymbol{B}_{0}$ and normal to the boundary should both be continuous [30]. However, this adds the complication of solving the Maxwell equations in the surrounding vacuum. Previous research has shown $[14,17,20]$ that the eigenmodes are localized inside the plasma (albeit close to the outboard edge) by a well structure determined by the equilibrium magnetic field and density. The perturbation in the vacuum region is therefore not expected to have a large influence on the mode structure inside the plasma, so the present implementation is restricted to solution of the eigenmode equations inside the plasma.

A boundary condition is still needed though, and an ideal conducting wall at the plasma edge would be a natural choice, not because it is necessarily similar to the configuration in existing spherical tokamaks, but because it would ensure that no energy flows in or out of the plasma. This boundary condition, however, leads to unphysical solutions partially located directly adjacent to the boundary [31] which dominate the spectrum and make it very difficult to find any internally localized modes. In contrast to [32], where global eigenmodes are sought with one reflection point caused by plasma inhomogeneity and the other reflection point being at the wall, we are here looking for modes that are contained solely by the plasma inhomogeneity, as in e.g. [33]. The boundary condition should only affect a small evanescent tail of the eigenmode that spreads beyond the reflection point to the periphery.

The boundary condition that is used in the following is instead that the dominant magnetic component for the compressional wave, $b_{\|}$, vanishes at the edge. This corresponds to the plasma - vacuum boundary condition that $b_{\|}$should be continuous if one assumes that the magnetic perturbation is zero in the vacuum. It is a sufficient condition for the eigenmode equations since $b_{\|}$is the only component for which (A.1) (A.3) contain second order radial derivatives. Furthermore, the numerically calculated eigenmodes in Figure 3 confirm that $b_{\|}=0$ is a good boundary condition in the sense that the frequency and localization of the eigenmodes are less affected by where the edge of the domain is located than by where the density drops in the plasma. As shall be seen later, the Poynting vector is also very small at the boundary and tangential to it.

Numerically, there is also a "boundary" at $r=0$, i.e. on the magnetic axis. This point needs special treatment because some metric tensor elements, e.g. $g^{\theta \theta}$, diverge as $r \rightarrow 0$. Moreover, $b_{\|}$is continuous at $r=0$, but $b_{r}$ and $b_{\wedge}$ in general retain a $\theta$ dependence as $r \rightarrow 0$. To resolve this, a calculation point is placed on the magnetic axis for the three unknowns $B_{R}, B_{Z}$ and $B_{\phi}$, which are all continuous. Here, $R$ is the major radius, $Z$ is the vertical coordinate and $\boldsymbol{B}_{1}=B_{R} \nabla R+B_{Z} \nabla Z+B_{\phi} \nabla \phi$. For this point, the $\nabla R, \nabla Z$ and $\nabla \phi$ components of (4) are solved, and $B_{R}, B_{Z}$ and $B_{\phi}$ are linked by 

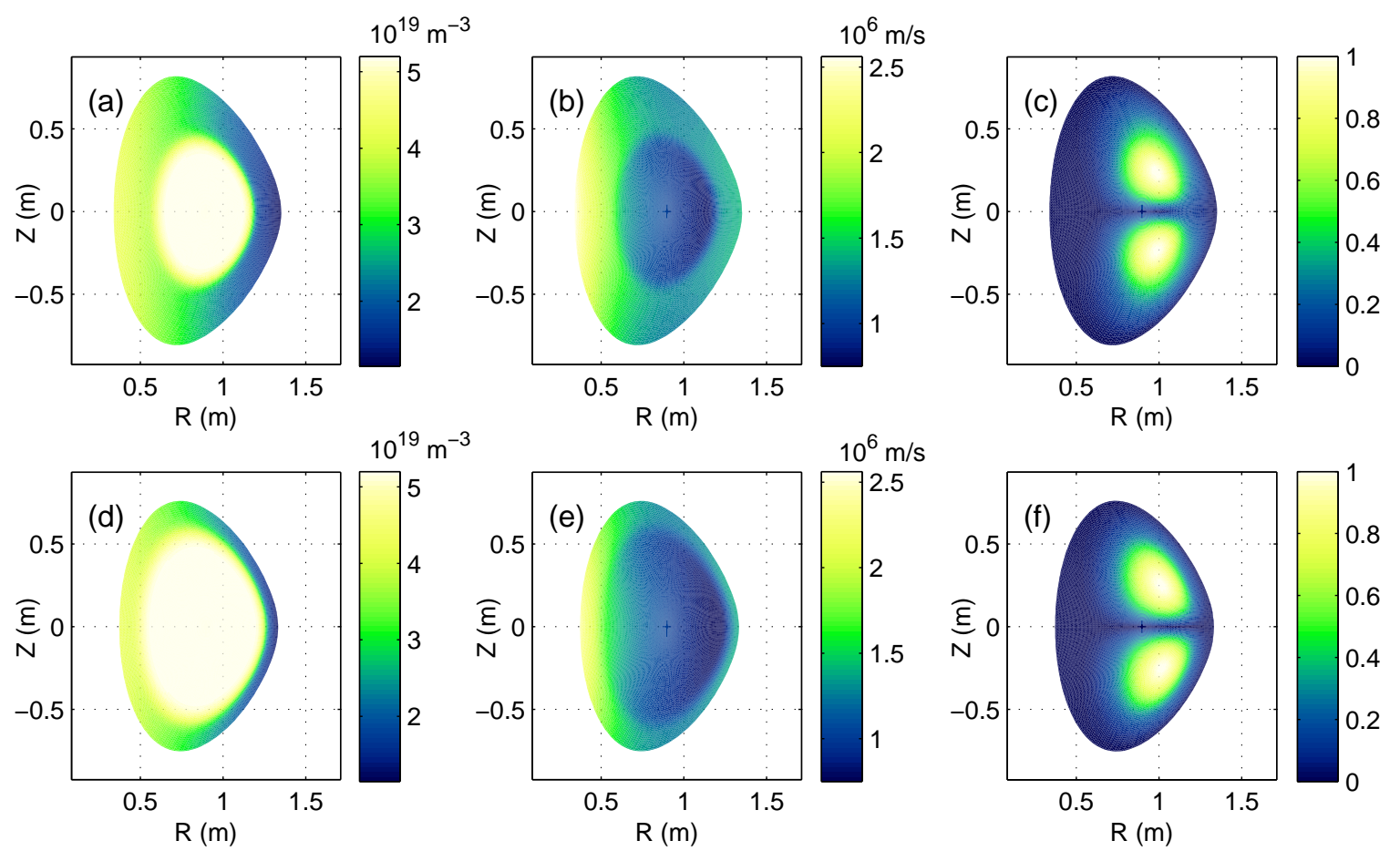

Figure 3. Top: Test case with narrow density profile and large computational domain for $n=5$ yielding $f=1.55 \mathrm{MHz}$ : (a) Density, (b) Alfvén speed, (c) $\left|B_{\|}\right|$. Bottom: (d)(e) Same as above for a test case with wider density profile and smaller computational domain yielding $f=1.42 \mathrm{MHz}$. The maxima in (f) are $10 \%$ farther apart than in (c). The edge density is chosen to be higher on the inboard than on the outboard side to avoid numerical difficulties with too large inboard Alfvén velocities caused by the high inboard magnetic field.

coordinate transformation matrices to the $b_{\|}, b_{r}$ and $b_{\wedge}$ components.

\section{Results and discussion}

To benchmark the numerical solver a comparison has been performed with results in [22], which were obtained for a circular plasma with aspect ratio $\epsilon^{-1}=3$, on-axis magnetic field $B_{0}=3.4 \mathrm{~T}$ and minor radius $a=1 \mathrm{~m}$. In [22] the mode frequency $51.7 \mathrm{MHz}$ is fixed and the density emerges from the calculation as an eigenvalue. As an example, an $n=1$ eigenmode with 7 wavelengths in the poloidal direction was found to have the eigendensity $3.42 \cdot 10^{18} \mathrm{~m}^{-3}$. For this density, the present numerical solver finds a mode with a similar structure shown in Figure 4(a)-(c) and the eigenfrequency $50.3 \mathrm{MHz}$. The small difference in eigenmode frequency can be attributed to the sensitivity of the solution to the radial profile of the density, which is assumed to be constant in the inner region and fall off at $r=1 \mathrm{~m}$ as shown in Figure 4(d). For this calculation the magnetic field was not obtained from an equilibrium solver, but was instead prescribed to be purely toroidal and vary as $1 / R$. Comparison with two other examples in [22] presented in Figures 4(e) and (f) also gives good agreement, so one can be confident 

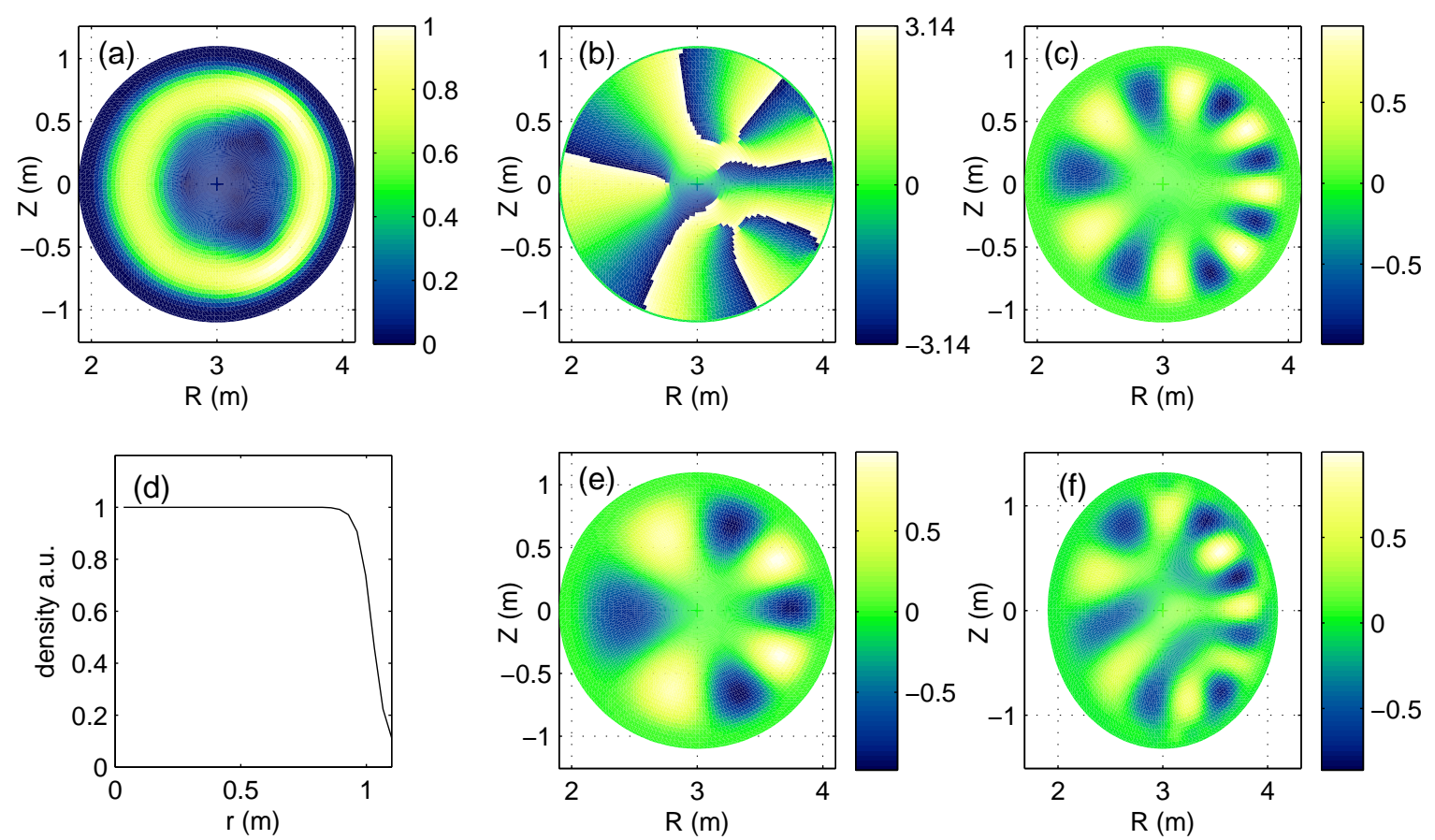

Figure 4. A benchmark with the results in [22]. Top: A solution for the density $3.423 \cdot 10^{18} \mathrm{~m}^{-3}$ with $f=50.3 \mathrm{MHz}$. (a) Absolute value, (b) complex phase and (c) real part of $B_{\|}$. Bottom: (d) The density profile. (e) A solution for the density $1.5784 \cdot 10^{18} \mathrm{~m}^{-3}$ with $f=50.2 \mathrm{MHz}$. (f) A solution for ellipticity $\kappa=1.2$ and density $2.7967 \cdot 10^{18} \mathrm{~m}^{-3}$ with $f=50.5 \mathrm{MHz}$.

that our numerical solver works for large aspect ratio tokamaks.

Having thus benchmarked the code, we now want to take one step further than in [22], and study highly shaped, tight aspect ratio equilibria. In the following, a typical MAST equilibrium calculated by the CHEASE code [24] is therefore used with the basic parameters $a=0.58 \mathrm{~m}, B_{0}=0.47 \mathrm{~T}$ on axis, $I=790 \mathrm{kA}$ and the density profile in Figure 3(d). The ion cyclotron frequency varies in this equilibrium from $2.4 \mathrm{MHz}$ on the outboard side to $7.4 \mathrm{MHz}$ on the inboard side. Figure 5 shows an example of a solution for the MAST equilibrium. Note that the the parallel component in Figure 5(c) dominates over the two perpendicular components in (a) and (b), and that the Poynting vector in Figure 5(d) is tangential to the boundary as previously mentioned. The eigenmodes obtained for the MAST equilibrium have a more standing wave-like structure than the ones in Figure 4. This can be seen by noting that the complex phase $\operatorname{Im}\left[\log \left(B_{\|}\right)\right]$of the parallel magnetic field component does not vary significantly on the scale of the amplitude variation for the parallel component in Figure 5(e). There is only a change from 0 to $\pi$ where $B_{\|}$goes through a zero and changes sign. Compare with Figure 4(b), which displays a gradual change of the phase as one go around one poloidal turn. This indicates a travelling wave, which has a monotonously increasing phase in a certain spatial direction (the direction of the local wave vector). In the toroidal direction the eigenmode is always travelling $\left(k_{\phi}=n / R\right)$, so the terms travelling 

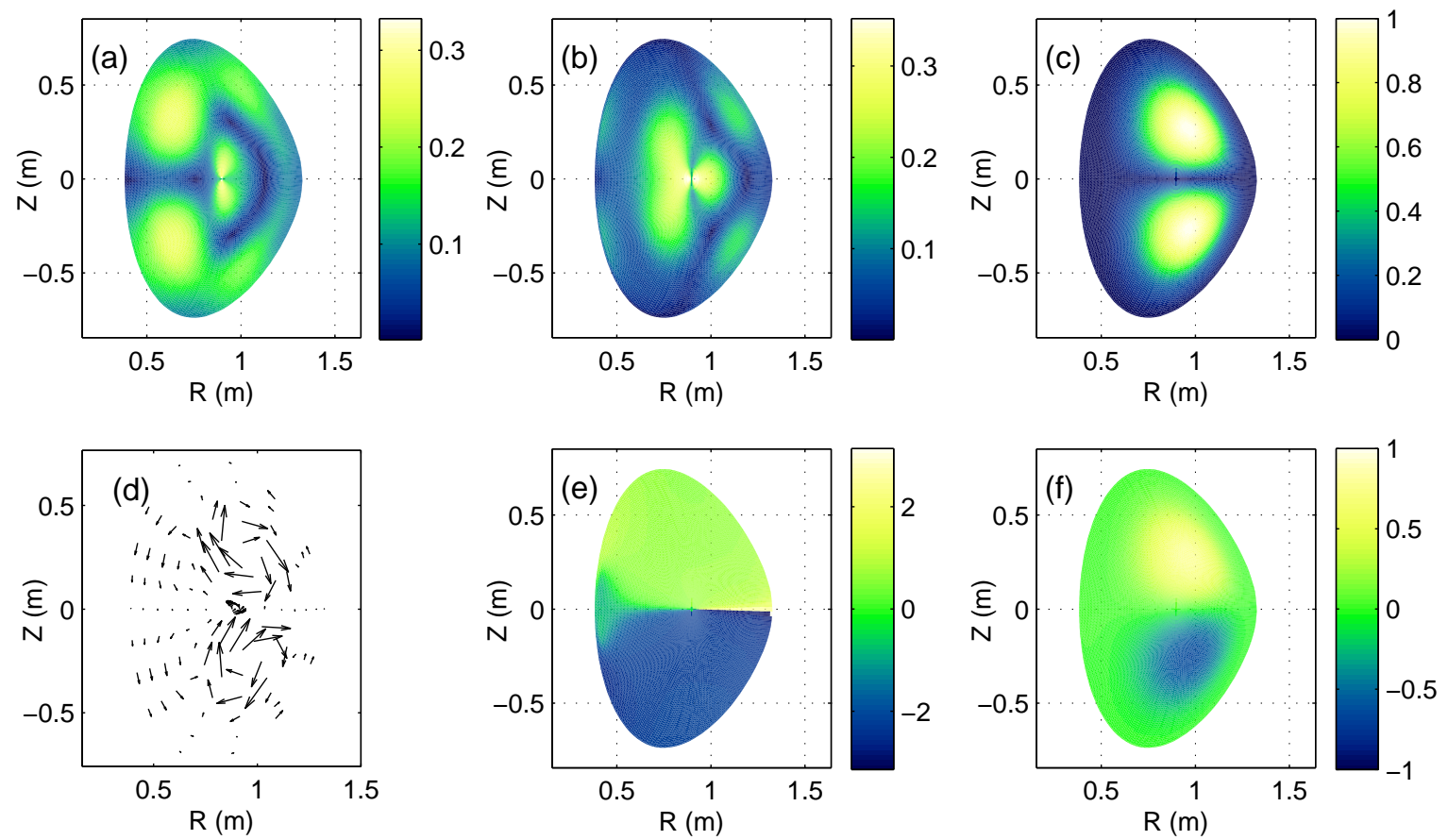

Figure 5. Example of an $n=3$ eigenmode for the MAST equilibrium with $f=1.29 \mathrm{MHz}$. Top plots: (a) $\left|B_{r}\right|$, (b) $\left|B_{\wedge}\right|$ and (c) $\left|B_{\|}\right|$. Bottom plots: (d) the Poynting vector, (e) the complex phase of $B_{\|}$and (f) the real part of $B_{\|}$. Plots (a), (b), (c) and (f) are normalized to $\max \left(\left|B_{\|}\right|\right)$.

and standing wave-like here only refers to the directions perpendicular to $\nabla \phi$.

The validity of the assumption (9) is investigated a posteriori and it is found that it holds in almost the whole cross section, except near the inboard edge. Since it holds on the outboard side where the eigenmodes are localized, the solutions are expected to be approximately valid. The finding that $k_{\|}^{2}$ is comparable to $\omega^{2} / v_{\mathrm{A}}^{2}$ on the inboard side indicates that the eigenmodes can in reality have a more shear wave-like behaviour there. Indeed, calculations of the polarization show that for positive $n$ the perturbation is right-handed (the electron gyration direction) in the region of localization, but left handed (the ion gyration direction) close to the inboard edge. For negative $n$, the solutions are most often right-handed in the whole cross section.

Figure 6 presents the lowest order different eigenmode structures, and the eigenfrequencies for these structures and different toroidal mode numbers $n$ are shown in Figure 7. One would expect from the simplest dispersion relation $\omega^{2}=v_{\mathrm{A}}^{2} k^{2}=$ $v_{\mathrm{A}}^{2}\left(n^{2} / R^{2}+k_{r}^{2}+k_{\theta}^{2}\right)$ that if the radial and poloidal wavenumbers $k_{r}$ and $k_{\theta}$ were independent of $n$, then the relation between $f$ and $n$ should be hyperbolic. For the high $k_{r}^{2}$ and $k_{\theta}^{2}$ eigenmode structures in Figure 7 (e.g. the one denoted by left pointing triangles) the the relation $f(n)$ in deed has a positive discrete second derivative. However, for most of the mode structures the relation $f(n)$ appears to be closer to an affine function than a hyperbola. The reason is that the potential well associated with the effective potential $V \equiv n^{2} / R^{2}-\omega^{2} / v_{\mathrm{A}}^{2}[23]$ becomes more concentrated to the 


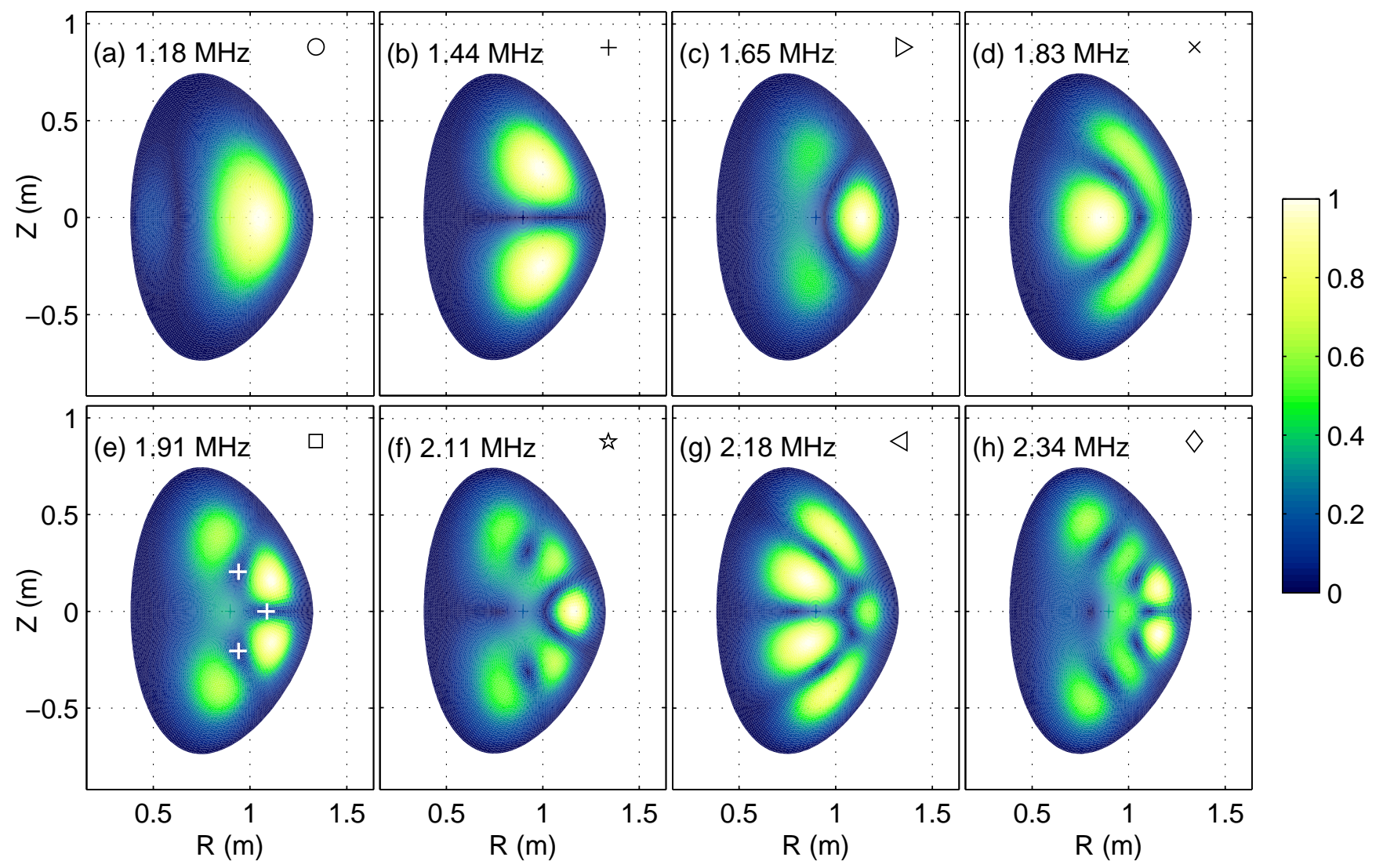

Figure 6. Different eigenmode structures for the MAST equilibrium for $n=5$. The crosses in (e) mark locations where $\left|B_{\|}\right|=0$.

outboard side with increasing $|n|$, and therefore the eigenmodes become more and more localized, i.e. $k_{r}$ and $k_{\theta}$ increase with $|n|$. This was reported in [20] and is also seen in calculations with the present numerical solver.

Moreover, it is found for a fixed $n$ that with higher frequency the solutions change from a simple standing wave character to a more complicated form. As an example, the complex phase of $B_{\|}$for the solution in Figure 6(e) is given in Figure 8. As time progresses, the extrema of $\operatorname{Re}\left(B_{\|} e^{-i \omega t}\right)$ circle around the $\left|B_{\|}\right|=0$ points marked with white crosses in both figures. In the theories of [20] and [17] it was assumed that the solution is a travelling wave $\propto e^{i n q \theta}$ in the poloidal direction, which is not a suitable representation of the more standing wave-like behaviour of the lowest order eigenmodes computed here.

These results point at the differences between the eigenmodes found in the MAST geometry and in larger aspect ratio circular cross sections [as e.g. in Figure 4(a)(e)]. However, it is seen in Figure 9, that when the geometry is varied from a large aspect ratio circular plasma to a low aspect ratio elongated plasma the transition in the eigenmode structure is gradual. The lowering of the the aspect ratio causes an increase in the relative variation in $B_{0}$ across the cross section, which leads to a stronger localization on the outboard side. The decrease in the aspect ratio and the increase in 


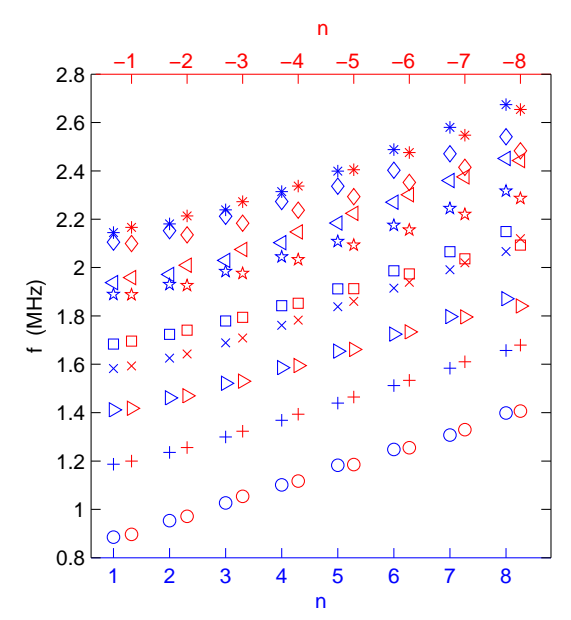

Figure 7. Eigenmode frequencies for different $n$. For clarity, the top axis for negative $n$ is slightly shifted to the right compared with the bottom axis for positive $n$. The different symbols correspond to the different eigenmode structures shown in Figure 6.

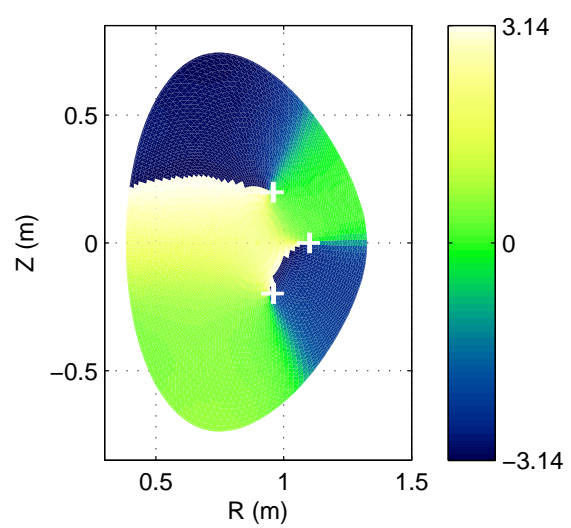

Figure 8. The complex phase of $B_{\|}$for the solution in Figure 6(e). The crosses mark locations where $\left|B_{\|}\right|=0$.

the elongation both distort the simple poloidally travelling wave property of the mode. The gradual transition from large to small aspect ratio is especially interesting in view of the similarities between experimental observations of CAEs in DIII-D (with aspect ratio $\epsilon^{-1}=2.9$ ) and the spherical tokamak NSTX (with aspect ratio $\epsilon^{-1}=1.3$ ) [15]. Note however that the flat central density profile studied here may not be representative for those experiments [23].

Based on the findings in Figure 9, we propose a classification scheme for spherical tokamak eigenmodes, in which the eigenmode structure is followed in a series of calculations for magnetic configurations with gradually increasing aspect ratio and decreasing elongation. The spherical tokamak eigenmode is then classified by the mode numbers of the obtained large aspect ratio eigenmode. The topology of the solutions in Figures $9(\mathrm{~d})$ and $(\mathrm{h})$ is the same as in Figures 6(d) and (e), although the frequencies 

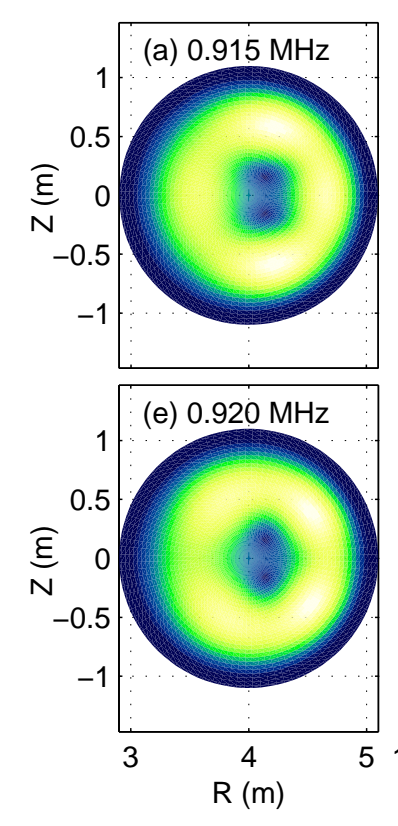
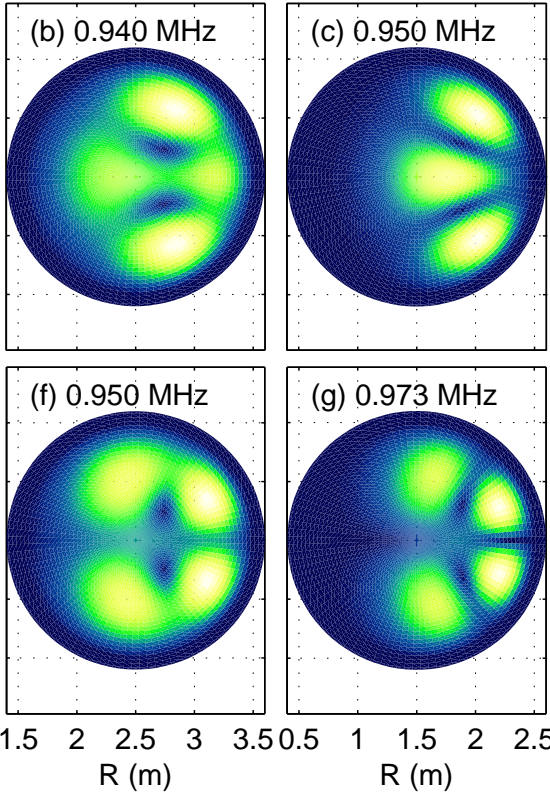

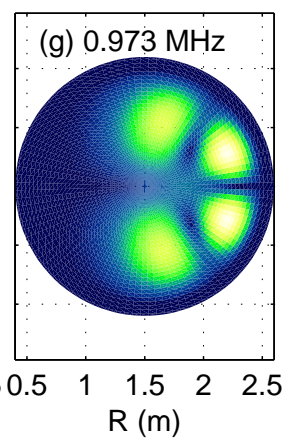

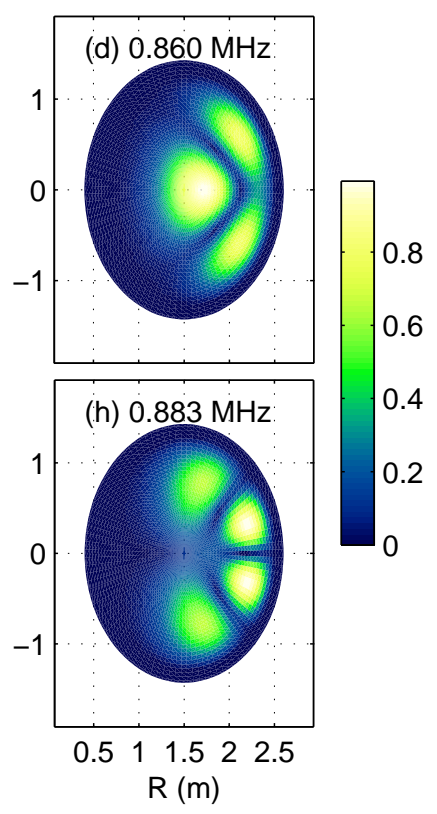

Figure 9. $\left|B_{\|}\right|$for two different types of eigenmode (rows) in four simple magnetic configurations (columns). In all cases the central density profile is flat as in Figure 4 with $n_{\mathrm{e}}=5 \cdot 10^{19} \mathrm{~m}^{-3}$ on axis, $B_{0}=0.5 \mathrm{~T}$ on axis and $B_{0} \propto 1 / R$. The aspect ratio decreases from left to right in the first three columns, and the elongation is higher in the last column. Figures (d) and (h) correspond to the mode structures in Figures 6(d) and (e).

and the cross section geometries are somewhat different. The corresponding large aspect ratio modes in Figures 9(a) and (e) have one anti-node in the radial direction, so the radial mode number is $s=1$. By inspection of the complex phase of the solutions in Figures 9(a) and (e) (not shown), the two large aspect ratio eigenmodes can be classified as $m=2$ and $m=-2$ modes, respectively. In the same way, each mode in Figure 6 can be classified in terms of the radial and poloidal mode numbers $s$ and $m$ of the corresponding large aspect ratio eigenmode, where $s$ denotes the number of oscillations between $r=0$ and the edge. The result for Figures 6(a)-(h) is (a) $s=1 / 2, m=0$, (b) $s=1, m=1$, (c) $s=1, m=-1$, (d) $s=1, m=2$, (e) $s=1, m=-2$, (f) $s=3 / 2, m=0,(\mathrm{~g}) s=1, m=3$ and (h) $s=2, m=1$. The classification scheme proposed here differs from the one in [23], which is directly related to the small aspect ratio solution. The present classification scheme is instead related to an equivalent large aspect ratio eigenmode, and it is in general nontrivial to deduce the values of $s$ and $m$ just by studying the spherical tokamak eigenmode itself.

The obtained eigenfrequencies for MAST in Figure 7 are not expected to exactly match the experimental observations, because they are sensitive to details of the equilibrium, especially the density profile. For comparison with experiments, theory and other numerical works it is therefore better to study the frequency difference between eigenmodes of different structures in the poloidal cross section or between different 
toroidal mode numbers. The frequency splitting between the first two eigenmode structures in Figure 6 (marked with circles and crosses, respectively) is in the range $260-310 \mathrm{kHz}$. Between the second and third (marked with crosses and triangles), the difference is $220-230 \mathrm{kHz}$, and between the third and fourth (triangles and $\mathrm{x}$-es), $170-180 \mathrm{kHz}$. This range of frequency splittings is consistent with the experimentally observed frequency differences between modes of the same $n$ in MAST [13]. They are also in general agreement with the poloidal eigenmode frequency splitting found theoretically [17, 20] and numerically [23] for NSTX, which is a spherical tokamak with an equilibrium not very different from MAST.

The frequency splitting between successive $n$ in Figure 7 is $\simeq 40-70 \mathrm{kHz}$, which is in the same range as was obtained with the ideal MHD code NOVA [23] for NSTX. The convention in this paper is that $\boldsymbol{B}_{0} \cdot \nabla \phi>0$ and $\boldsymbol{J}_{0} \cdot \nabla \phi<0$ and that perturbations are proportional to $\exp (-\mathrm{i} n \phi-\mathrm{i} \omega t)$, which implies that $n>0$ corresponds to co- $\boldsymbol{J}_{0}$ (counter- $\boldsymbol{B}_{0}$ ) propagation.

The eigenmode equations solved in this work include the Hall term and the equilibrium current, and it is therefore interesting to see how these terms influence the calculated eigenmodes. The eigenmodes for negative $n$ have a similar structure to the modes for the corresponding positive $n$, but there is a small difference in frequency. The negative $n$ frequencies in Figure 7 are slightly shifted compared with the positive ones, a difference which disappears if the Hall term is switched off by setting $F$ to zero. If the Hall term is included in the eigenmode equation but not the $\boldsymbol{J}_{0}$ terms, then the $n>0$ frequencies are $50-100 \mathrm{kHz}$ higher than the corresponding $n<0$ frequencies. When $\boldsymbol{J}_{0}$ is also included, the last term in (4) has the opposite effect that the positive $n$ mode frequencies typically become shifted downwards $\sim 30 \mathrm{kHz}$ and the negative $n$ modes are shifted upwards $\sim 30 \mathrm{kHz}$. On the whole, the frequency difference between plus and minus $n$ in Figure 7 with all effects included is generally smaller than $50 \mathrm{kHz}$, and it has different signs for different eigenmodes. Moreover, for an equilibrium with $\boldsymbol{B}_{0} \cdot \boldsymbol{J}_{0}>0$ the frequency difference between plus and minus $n$ has the opposite sign compared with the $\boldsymbol{B}_{0} \cdot \boldsymbol{J}_{0}<0$ equilibrium discussed above.

In [20], edge localized eigenmodes were obtained, for which there was an asymmetry with respect to the sign of the mode number $n$ of the existence of solutions. This asymmetry is not found here, because the eigenmodes are localized farther in from the outboard edge, a result which was also reported using the theory of [20] for a realistic MAST equilibrium in [14]. The eigenmodes are radially more extended and have their maximum at around $r \sim a / 2$, and the frequency is therefore not as close to the ion cyclotron frequency as in [20], so the Hall term is less important. The ion cyclotron frequency in this equilibrium is around $3 \mathrm{MHz}$ where the eigenmodes are located. In the MAST experiments, modes of both signs of $n$ were observed [14], although negative toroidal mode numbers were more commonly seen [13], an asymmetry which is not seen in the present work and which is therefore likely to be due to the excitation by the energetic ions. 


\section{Conclusions}

Two-dimensional eigenmode structures for CAE below the ion cyclotron frequency have been calculated numerically using the cold plasma Hall-MHD equations for a spherical tokamak equilibrium. The influence of the equilibrium current and the Hall terms in the eigenmode equation was studied, and it was found that including the Hall term removes the degeneracy of eigenmodes which differ only in the sign of $n$. It shifts the $n>0$ modes up and the $n<0$ modes down in frequency for an equilibrium with $\boldsymbol{B}_{0} \cdot \boldsymbol{J}_{0}<0$. When the equilibrium current is also included this frequency difference between plus and minus $n$ is reduced to less than $50 \mathrm{kHz}$. For the MAST equilibrium studied here, the Hall term was not found to have any effect on the existence of eigenmodes for different signs of $n$, because the eigenmodes are not localized as close to the outboard edge as in [20]. The frequency splitting between the first few low order eigenmode structures is around $200-300 \mathrm{kHz}$, and for higher orders the frequencies are more closely spaced. The lowest order eigenmodes have a standing wave-like structure, but the higher order eigenmodes show a complicated behaviour which locally resembles small eddies of travelling waves. A classification method is proposed which relates each spherical tokamak eigenmode to the three mode numbers $n, m$ and $s$ of the corresponding large aspect ratio circular cross section eigenmode obtained by gradually decreasing the elongation and increasing the aspect ratio.

\section{Acknowledgments}

The authors want to thank Yann Camenen for support with the CHEASE code and Sergei Sharapov, Matthew Lilley, Simon Pinches and Lynton Appel for helpful discussions. This work was funded by the UK Engineering and Physical Sciences Research Council.

\section{Appendix}

The component of the eigenmode equation (4) along $\nabla r$ is

$$
\begin{aligned}
& \omega^{2} g^{r r} b_{r}=\left(\boldsymbol{B}_{0} \cdot \nabla\right) \mathrm{i} F(\boldsymbol{\Lambda} \cdot \nabla) b_{\|}+(\boldsymbol{B} \cdot \nabla)\left[G B_{0}^{2} \nabla r \cdot \nabla b_{\|}-2 G b_{\|} \tilde{p}^{\prime} g^{r r}\right]+ \\
& -\left(\boldsymbol{B}_{0} \cdot \nabla\right) \mathrm{i} F\left(\boldsymbol{B}_{0} \cdot \nabla\right) g^{r r} b_{\wedge}+\left(\boldsymbol{B}_{0} \cdot \nabla\right) G b_{\wedge}\left(\boldsymbol{\Lambda} \cdot \boldsymbol{H}+g^{r r} \alpha B_{0}^{2}\right)+ \\
& -\left(\boldsymbol{B}_{0} \cdot \nabla\right) G g^{r r}\left(\boldsymbol{B}_{0} \cdot \nabla\right) b_{r}-\alpha\left(\boldsymbol{B}_{0} \cdot \nabla\right) \mathrm{i} F g^{r r} b_{r}-\tilde{p}^{\prime} B_{0}^{-2}(\boldsymbol{\Lambda} \cdot \nabla) \mathrm{i} F g^{r r} b_{r},
\end{aligned}
$$

where $\tilde{\boldsymbol{J}}_{0} \equiv \alpha \boldsymbol{B}_{0}+\tilde{p}^{\prime} B_{0}^{-2} \boldsymbol{\Lambda}$ defines $\alpha$ and $\tilde{p}^{\prime}$, and where $\boldsymbol{H} \equiv \nabla \times \boldsymbol{\Lambda}=-2 \boldsymbol{D}+\alpha \boldsymbol{\Lambda}+$ $\left(\nabla^{2} r-\tilde{p}^{\prime} g^{r r} / B_{0}^{2}\right) \boldsymbol{B}_{0}$ and $\boldsymbol{D} \equiv\left(\boldsymbol{B}_{0} \cdot \nabla\right) \nabla r$. The component of (4) along $\boldsymbol{\Lambda}$ is

$$
\begin{aligned}
& \omega^{2} g^{r r} B_{0}^{2} b_{\wedge}=b_{r}\left[\mathrm{i} F \alpha \boldsymbol{H} \cdot \boldsymbol{\Lambda}+\mathrm{i} F \tilde{p}^{\prime} g^{r r} B_{0}^{-2}\left(\tilde{p}^{\prime} g^{r r}+2 \boldsymbol{B}_{0} \cdot \boldsymbol{D}\right)+\nabla r \cdot \nabla\left(\mathrm{i} F \tilde{p}^{\prime} g^{r r}\right)\right]+ \\
& +\mathrm{i} F \tilde{p}^{\prime} g^{r r} \nabla r \cdot \nabla b_{r}+g^{r r}\left(\boldsymbol{B}_{0} \cdot \nabla\right) \mathrm{i} F\left(\boldsymbol{B}_{0} \cdot \nabla\right) b_{r}+ \\
& -g^{r r}\left(\boldsymbol{B}_{0} \cdot \nabla\right) G \alpha B_{0}^{2}-2 G \boldsymbol{\Lambda} \cdot \boldsymbol{D}\left(\boldsymbol{B}_{0} \cdot \nabla\right) b_{r}+ \\
& b_{\wedge}\left[\mathrm{i} F \alpha B_{0}^{2}\left(\boldsymbol{B}_{0} \cdot \nabla\right) g^{r r}+\left(\boldsymbol{B}_{0} \cdot \nabla\right) 2 \mathrm{i} F \boldsymbol{\Lambda} \cdot \boldsymbol{D}\right]+\mathrm{i} F \boldsymbol{\Lambda} \cdot(\boldsymbol{H}-2 \boldsymbol{D})\left(\boldsymbol{B}_{0} \cdot \nabla\right) b_{\wedge}+
\end{aligned}
$$




$$
\begin{aligned}
& +b_{\wedge}\left[G B_{0}^{2}\left(4\left|\boldsymbol{D}_{\perp}\right|^{2}-2 \alpha \boldsymbol{\Lambda} \cdot \boldsymbol{D}\right)-\left(\boldsymbol{B}_{0} \cdot \nabla\right) G B_{0}^{2}\left(\boldsymbol{B}_{0} \cdot \nabla\right) g^{r r}\right]+ \\
& -g^{r r}\left(\boldsymbol{B}_{0} \cdot \nabla\right) G B_{0}^{2}\left(\boldsymbol{B}_{0} \cdot \nabla\right) b_{\wedge}-G B_{0}^{2}\left(\boldsymbol{B}_{0} \cdot \nabla g^{r r}\right)\left(\boldsymbol{B}_{0} \cdot \nabla\right) b_{\wedge}+ \\
& +\left(2 \mathrm{i} F \tilde{p}^{\prime} g^{r r} \boldsymbol{B}_{0}-\mathrm{i} F B_{0}^{2} \boldsymbol{H}_{\perp}\right) \cdot \nabla b_{\|}+2 b_{\|} g^{r r}\left(\boldsymbol{B}_{0} \cdot \nabla\right) \mathrm{i} F \tilde{p}^{\prime}-\left(\boldsymbol{B}_{0} \cdot \nabla\right) \mathrm{i} F B_{0}^{2} \nabla r \cdot \nabla b_{\|}+ \\
& +2 G \boldsymbol{H} \cdot \boldsymbol{\Lambda} \tilde{p}^{\prime} b_{\|}+G B_{0}^{2} \boldsymbol{B}_{0} \times \boldsymbol{H} \cdot \nabla b_{\|}+\left(\boldsymbol{B}_{0} \cdot \nabla\right) G B_{0}^{2} \boldsymbol{\Lambda} \cdot \nabla b_{\|} .
\end{aligned}
$$

Finally, the component of (4) along $\boldsymbol{B}_{0}$ is

$$
\begin{aligned}
& \omega^{2} B_{0}^{2} b_{\|}=-(\boldsymbol{\Lambda} \cdot \nabla) \mathrm{i} F\left(\boldsymbol{B}_{0} \cdot \nabla\right) b_{r}+b_{r} \mathrm{i} F \alpha B_{0}^{2} \nabla^{2} r+\nabla r \cdot \nabla\left(\mathrm{i} F \alpha B_{0}^{2} b_{r}\right)+ \\
& +\left(\nabla^{2} r\right) G B_{0}^{2}\left(\boldsymbol{B}_{0} \cdot \nabla\right) b_{r}+(\nabla r \cdot \nabla) G B_{0}^{2}\left(\boldsymbol{B}_{0} \cdot \nabla\right) b_{r}+\boldsymbol{\Lambda} \cdot \nabla\left(G B_{0}^{2} \alpha b_{r}\right)+\tilde{p}^{\prime} G g^{r r} \boldsymbol{B}_{0} \cdot \nabla b_{r}+ \\
& +b_{\wedge}\left[2 \nabla \cdot\left(\mathrm{i} F B_{0}^{2} \boldsymbol{D}_{\perp}\right)+\mathrm{i} F \tilde{p}^{\prime}\left(\boldsymbol{B}_{0} \cdot \nabla\right) g^{r r}\right]+\mathrm{i} F\left(B_{0}^{2} \nabla^{2} r+\tilde{p}^{\prime} g^{r r}\right) \boldsymbol{B}_{0} \cdot \nabla b_{\wedge}+ \\
& +(\nabla r \cdot \nabla) \mathrm{i} F B_{0}^{2}\left(\boldsymbol{B}_{0} \cdot \nabla\right) b_{\wedge}+2 \mathrm{i} F B_{0}^{2} \boldsymbol{D}_{\perp} \cdot \nabla b_{\wedge}+b_{\wedge} G B_{0}^{2} \nabla \cdot\left(2 \boldsymbol{B}_{0} \times \boldsymbol{D}\right)+ \\
& b_{\wedge}\left(2 \boldsymbol{B}_{0} \times \boldsymbol{D}\right) \cdot \nabla G B_{0}^{2}-2 b_{\wedge} G \tilde{p}^{\prime} \boldsymbol{\Lambda} \cdot \boldsymbol{D}+(\boldsymbol{\Lambda} \cdot \nabla) G B_{0}^{2}\left(\boldsymbol{B}_{0} \cdot \nabla\right) b_{\wedge}+ \\
& +G B_{0}^{2}\left(2 \boldsymbol{B}_{0} \times \boldsymbol{D}\right) \cdot \nabla b_{\wedge}+\left(\nabla \mathrm{i} F B_{0}^{2} \times \boldsymbol{B}_{0}\right) \cdot \nabla b_{\|}+\mathrm{i} F B_{0}^{2} \alpha \boldsymbol{B}_{0} \cdot \nabla b_{\|}-2 b_{\|} \boldsymbol{\Lambda} \cdot \nabla\left(\mathrm{i} F \tilde{p}^{\prime}\right) \\
& -\mathrm{i} F \tilde{p}^{\prime} \boldsymbol{\Lambda} \cdot \nabla b_{\|}-\nabla \cdot G B_{0}^{4} \nabla_{\perp} b_{\|}+2 b_{\|} G B_{0}^{2} \tilde{p}^{\prime} \nabla^{2} r+b_{\|} \nabla r \cdot \nabla\left(2 G B_{0}^{2} \tilde{p}^{\prime}\right)+ \\
& +G B_{0}^{2} \tilde{p}^{\prime} \nabla r \cdot \nabla b_{\|}+2 G \tilde{p}^{\prime 2} g^{r r} b_{\|} .
\end{aligned}
$$

From these equations and the assumption (9), the coefficients of (14) are derived using co- and contravariant representations of the vectors in the $r, \theta, \phi$ coordinate system. For instance, the $\left(\boldsymbol{B}_{0} \cdot \nabla\right) X$ operator becomes $B_{0}^{\theta} \partial X / \partial \theta-i n B_{0}^{\phi} X$ if $X$ is a perturbed quantity and $B_{0}^{\theta} \partial X / \partial \theta$ if $X$ is an equilibrium quantity.

\section{References}

[1] Gates D A, Gorelenkov N N and White R B 2001 Ion heating by fast-particle-induced Alfvén turbulence Phys. Rev. Lett. 87205003

[2] Mahajan S M and Ross D W 1983 Spectrum of compressional Alfvén waves Phys. Fluids 262561

[3] Coppi B, Cowley S, Kulsrud R, Detragiache P and Pegoraro F 1986 High-energy components and collective modes in thermonuclear plasmas Phys. Fluids 294060

[4] Coppi B 1993 Origin of radiation emission induced by fusion reaction products Phys. Lett. A 172 439

[5] Fülöp T, Kolesnichenko Y I, Lisak M and Anderson D 1997 Origin of ion cyclotron emission in tokamaks Nucl. Fusion $\mathbf{3 7} 1281$

[6] Gorelenkov N N and Cheng C Z 1995 Alfvén cyclotron instability and ion cyclotron emission Nucl. Fusion 351743

[7] Fülöp T, Lisak M, Kolesnichenko Y I and Anderson D 2000 The radial and poloidal localization of fast magnetoacoustic eigenmodes in tokamaks Phys. Plasmas 71479

[8] Coppi B, Penn G and Riconda C 1997 Excitation of contained modes by high energy nuclei and correlated cyclotron emission Ann. Phys. 261117

[9] Penn G, Riconda C and Rubini F 1998 Description of contained mode solutions to the relevant magnetosonic-whistler wave equations Phys. Plasmas 52513

[10] Kolesnichenko Y I, Fülöp T, Lisak M and Anderson D 1998 Localized fast magnetoacoustic eigenmodes in tokamak plasmas Nucl. Fusion $\mathbf{3 8} 1871$

[11] McClements K G, Gryaznevich M P, Sharapov S E, Akers R J, Appel L C, Counsell G F, Roach C M and Majeski R 1999 Physics of energetic particle-driven instabilities in the START spherical tokamak Plasma Phys. Control. Fusion 41661 
[12] Fredrickson E D, Gorelenkov N, Cheng C Z, Bell R, Darrow D, Johnson D, Kaye S, LeBlanc B and Menard J 2001 Observation of compressional Alfvén modes during neutral-beam heating on the national spherical torus experiment Phys. Rev. Lett. 87145001

[13] Gryaznevich M et al 2008 Recent experiments on Alfvén eigenmodes in MAST Nucl. Fusion 48 084003

[14] Appel L C, Fülöp T, Hole M J, Smith H M, Pinches S D, Vann R G L and The MAST Team 2008 Compressional Alfvén eigenmodes on mast Plasma Phys. Control. Fusion 50115011

[15] Heidbrink W, Fredrickson E, Gorelenkov N, Rhodes T and Zeeland M V 2006 Observation of compressional Alfvén eigenmodes (CAE) in a conventional tokamak Nucl. Fusion 46324

[16] Gorelenkov N N, Cheng C Z, Fredrickson E D, Belova E, Gates D, Kaye S, Kramer G J, Nazikian R and White R 2002 Compressional Alfvén eigenmode instability in NSTX Nucl. Fusion 42977

[17] Gorelenkov N N, Cheng C Z and Fredrickson E 2002 Compressional Alfvén eigenmode dispersion in low aspect ratio plasmas Phys. Plasmas 93483

[18] Gorelenkov N N, Belova E, Berk H L, Cheng C Z, Fredrickson E, Heidbrink W W and Kramer G J 2004 Beam ion driven instabilities in the national spherical tokamak experiment Phys. Plasmas 112586

[19] Fredrickson E D, Gorelenkov N N and Menard J 2004 Phenomenology of compressional Alfvén eigenmodes Phys. Plasmas 113653

[20] Smith H, Fülöp T, Lisak M and Anderson D 2003 Localization of compressional Alfvén eigenmodes in spherical tori Phys. Plasmas 101437

[21] Gorelenkov N N, Fredrickson E D, Belova E, Gates D, Kaye S and White R 2003 Theory and observations of high frequency Alfvén eigenmodes in low aspect ratio plasmas Nucl. Fusion $\mathbf{4 3}$ 228

[22] Hellsten T and Laxåback M 2003 Edge localized magnetosonic eigenmodes in the ion cyclotron frequency range Phys. Plasmas 104371

[23] Gorelenkov N, Fredrickson E, Heidbrink W, Crocker N, Kubota S and Peebles W 2006 Discrete compressional Alfvén eigenmode spectrum in tokamaks Nuclear Fusion 46 S933

[24] Lütjens H, Bondeson A and Sauter O 1996 The CHEASE code for toroidal MHD equilibria Comp. Phys. Comm. 97219

[25] Hazeltine R D and Waelbroeck F L 1998 The Framework of Plasma Physics (Reading: Perseus Books)

[26] Villard L, Appert K, Gruber R and Vaclavik J 1986 Global waves in cold plasmas Comp. Phys. Rep. 495

[27] Cuperman S, Bruma C and Komoshvili K 2003 Solution of the resisitve two-fluid wave equations for Alfvénic modes in spherical tokamak plasmas J. Plasma Phys. 6915

[28] Cramer N F 2001 The Physics of Alfvén Waves (Berlin: Wiley-VCH) 1 ed.

[29] Arnoldi W E 1951 The principle of minimized iterations in the solution of the matrix eigenvalue problem Quart. J. Applied Math. 917

[30] Goedbloed H and Poedts S 2004 Principles of Magnetohydrodynamics (Cambridge: Cambridge University Press)

[31] Young N P, Sharapov S E, Nakariakov V M and JET EFDA contributors 2006 Identifying Alfvén eigenmodes in the early phase of advanced tokamak plasmas Plasma Phys. Control. Fusion $\mathbf{4 8}$ 295

[32] Itoh S I, Itoh K and Fukuyama A 1984 Beam-driven ICRF instability and associated nonclassical transport in tokamak Plasma Phys. Control. Fusion 261311

[33] Lilley M K and Sharapov S E 2007 Compressional Alfvén and ion-ion hybrid modes in the deuterium-tritium plasma of a spherical tokamak power plant Phys. Plasmas 14082501 